\title{
Atitudes parentais sobre Educação Física inclusiva
}

\author{
Celina Luísa Raimundo Martins \\ Doutora em Investigação em Atividade Física, Desporto e Saúde (Universidade de Vigo) \\ Pesquisadora na Universidade de Vigo \\ Porto, Portugal \\ celinammm@gmail.com \\ Margarita Rosa Pino Juste \\ Doutora em Pedagogia pela Universidade de Santiago de Compostela \\ Professora titular do Departamento de Didática, Organização Escolar \\ e Métodos de Investigação da Universidade de Vigo, Espanha \\ mpino@uvigo.es
}

Resumo As atitudes em relação à inclusão de estudantes com deficiência na escola regular assumem um papel decisivo na construção de uma sociedade democrática e inclusiva. O objetivo deste estudo foi descrever as atitudes parentais sobre a inclusão nas classes de Educação Física (EF). Aplicou-se uma escala de atitudes sociais a uma amostra de 213 pais ou tutores legais de estudantes portugueses. Para discussão dos resultados, constituiu-se um grupo de discussão composto por cinco professores de EF, dois pais e dois estudantes. Os resultados evidenciaram atitudes moderadamente positivas que se relacionaram com diversos fatores. Concluiu-se que o sucesso da inclusão requer que as famílias e a comunidade acreditem mais na competência das escolas, e que para tal são necessários mais recursos materiais e humanos, formação específica, diferenciação do curricular e trabalho colaborativo com as famílias e a comunidade.

Palavras-chave: atitudes, pais, educação inclusiva, educação física, necessidades educativas especiais.

\section{Introdução}

A o longo dos últimos anos, vem aumentando o interesse internaAcional e o compromisso em oferecer uma educação inclusiva de qualidade a todas as crianças com deficiência (Mantoan, 2007; Sassaki, 2007; Miles; Singal, 2010). Enquanto a educação especial se ocupa apenas com a educação de portadores de deficiência em instituições especializadas, a educação inclusiva veio ampliar a participação dessas pessoas nos estabelecimentos de ensino regular. Trata-se de uma reestruturação da cultura, da prática e das políticas vivenciadas nas escolas, de modo que estas respondam à diversidade de alunos. É uma abordagem mais humanística e democrática, que pretende atender as necessidades educativas dos alunos com deficiência, tendo como objetivo o seu desenvolvimento pessoal e social (Ainscow, 2002; Sassaki, 2007; Ruijs; Van Der Veen; Peetsma, 2010).

Um dos aspectos-chave da política da inclusão educativa tem sido a tendência cada vez mais comum para a Educação Física (EF) ser usada como um veículo importante na promoção da inclusão de estudantes com deficiência no ensino regular (Sherrill, 1998; Lieberman; James; Ludwa, 2004; Hutzler; Levi, 2008; Haycock; Smith, 2010; Qi; Há, 2012).

Se estamos verdadeiramente empenhados em melhorar a nossa sociedade global, é mais urgente do que nunca envolver efetivamente 
toda a comunidade no sucesso da educação inclusiva (Orsati; Causton-Theoharis, 2012). Desse modo, as atitudes sociais positivas de toda a comunidade escolar são fatores importantes para garantir experiências significativas de aprendizagem dos estudantes com deficiência (Block; Obrusnikova, 2007; Kalyva; Georgiadi; Tsakiris, 2007; Tripp; Rizzo; Webbert, 2007; Beckett, 2009).

Em face da importância do papel colaborativo das famílias na educação das crianças, torna-se crucial conhecer as suas atitudes, sendo esse conhecimento um ponto de partida para o desenvolvimento de relações de colaboração com a escola (Swart et al., 2004). Mas são poucos os estudos que abordam a forma como a inclusão em EF é percebida pelas famílias (MacDonald et al., 2004; Boer et al., 2012). Sendo assim, este estudo teve como objetivo principal aprofundar o conhecimento sobre as concepções que as famílias têm em relação à prática de EF inclusiva na escola regular e saber quais são os principais fatores que as influenciam.

\section{A formação social e a inclusão educacional}

O discurso sobre a inclusão aborda inevitavelmente a participação social das pessoas com deficiência em igualdade de oportunidades, destacando a importância de uma sociedade inclusiva (Beckett, 2009; Rocha et al., 2009). A integração na sociedade é considerada como fruto de um encontro de crenças sobre a própria identidade, como se fosse uma parte inata da essência de um indivíduo ou de uma determinada coletividade (Spreafico, 2009).

O conceito de pessoa com deficiência foi definido pela Organização Mundial de Saúde como a ausência ou a disfunção de uma estrutura psíquica, fisiológica ou anatômica que gere incapacidade para o desempenho de atividade, dentro do padrão considerado normal para o ser humano (OMS, 2007). Porém, a inclusão social dessas pessoas é muitas vezes limitada pelas barreiras atitudinais da sociedade, que impedem a sua participação plena e efetiva na sociedade em igualdade com os outros.

A problemática da inclusão é percebida mais como de natureza relacional, antes de ser um problema de direitos ou princípios (Swart et al., 2004; Sassaki, 2007; Orsati; Causton-Theoharis, 2012). Somos muitas vezes confrontados com diferentes discursos de exclusão. Uns salientam que a competitividade da nossa sociedade capitalista está associada à ideia de excelência, enquanto outros acentuam as dificuldades em concretizar as práticas inclusivas, já que estas exigem mudanças profundas na organização das instituições e nas atitudes de aceitação social. Neste sentido, uma resposta inclusiva eficaz pode traduzir-seno modo como uma sociedade se organiza para responder às necessidades das pessoas com deficiência, aceitando a diferença como enriquecedora e pertencente a si mesma.

A educação inclusiva é já uma política educacional de muitos países, apoiada por legislação própria e convertida em diretrizes na educação básica (Glat, 2004). De acordo com alguns autores (Ainscow, 2002; Sanches; Teodoro, 2012), a aceitação social é intimamente influenciada pela cultura das famílias, e esta e a escola são os dois fatores principais para a socialização de crianças e adolescentes. Assim, as atitudes presentes em ambos os contextos podem constituir-se como facilitadores ou inibidores de um bom desenvolvimento social de crianças e jovens com deficiência e da formação de atitudes e valores inclusivos de todos os estudantes.

A relevância do papel dos pais no processo de inclusão escolar já foi discutida na Declaração de Salamanca (artigos 57 a 60), que indicam que é uma tarefa a ser dividida entre pais e profissionais (Unesco, 1994). Também nesse documento é enfatizado que uma atitude positiva das famílias irá favorecer a integração escolar e social dos estudantes com deficiência, no entanto ressalta que elas precisam ser mais bem esclarecidas para que possam assumir uma participação mais ativa na educação dos seus filhos.

Rocha et al. (2009) acentuam que "a educação inclusiva é um projeto a ser construído por todos, família e população em geral e só terá êxito quando as atitudes em relação à inclusão escolar forem positivas" (p. 251). Outros autores confirmam que a dimensão relacional familiar éuma base fundamental do percurso inclusivo (Souza, Silva e Pontes, 2011; Correia; Serrano, 1997). Também a participação de toda a comunidade educativa é uma garantia de sucesso, na medida em que o envolvimento é mais enriquecido, conforme afirmam Pereira-Silva e Dessen (2007). Mas os autores enfatizam também o papel da família:

A família desempenha um papel tanto de impulsionadora como de inibidora dos processos de desenvolvimento do indivíduo [...]. Ela é a primeira mediadora entre o indivíduo e outros microssistemas e a cultura, transmitindo os significados culturais para os membros de seu grupo ao longo de gerações. (Pereira-Silva; Dessen, 2007, p. 430)

Em vários países, como no Brasil (Barbosa; Rosini; Pereira, 2007), na Grécia (Tafa; Manolitsis, 2003), na Índia (Singal, 2006) ou na Suécia (Göransson; Nilholm; Karlsson, 2011), vêm sendo discutidas criticamente as políticas de inclusão escolar, considerando a importância da cultura familiar. Também 
em Portugal, as famílias são consideradas como "elementos cruciais no planejamento, execução e avaliação dos programas de intervenção dos seus filhos" (Correia; Serrano, 2003, p. 61). Contudo, o fraco envolvimento parental que muitas vezes se verifica não decorre da falta de estímulo legal para a participação dos pais, como se pode concluir pela análise do artigo $3^{\circ}$ do Decreto-Lei n. ${ }^{\circ} 38$, de 7 de janeiro de 2008 (Portugal, 2008). Efetivamente, essa lei concede aos pais uma série de oportunidades de participação, nomeadamente:

- "os pais ou tutores têm o direito e o dever de participar ativamente, exercendo o poder paternal nos termos da lei, em tudo o que se relacione com a educação especial a prestar ao seu filho";

- "quando, comprovadamente, os pais ou tutores não exercerem o seu direito de participação, cabe à escola desencadear as respostas educativas adequadas em função das necessidades diagnosticadas";

- "quando os pais ou tutores não concordarem com as medidas educativas propostas pela escola, podem recorrer ao Ministério da Educação".

Com efeito, a família e a escola têm de conviver com os valores e as crenças transmitidos em ambos os contextos. Porém, os significados e atitudes sobre a inclusão dos estudantes com deficiência não são compartilhados igualmente por todas as pessoas, o que resulta em diferentes atitudes diante dos problemas (Melman, 2001). Emerge assim a necessidade de a proposta inclusiva ser amplamente discutida, aumentando a participação das famílias na escola.

\section{Atitudes parentais sobre inclusão}

A força das famílias, tal como a dos profissionais, é um fator-chave para progredir e alcançar uma educação de qualidade (Sassaki, 2007). Mortier et al. (2010) estudaram as experiências e perspectivas dos pais e professores que trabalharam na inclusão de estudantes com deficiência, destacando os benefícios das parcerias para aprofundar o conhecimento sobre essas crianças, permitindo assim melhorar o apoio prestado.

Nesse sentido, foram realizados estudos em diferentes países sobre a opinião dos pais sobre a inclusão de estudantes com deficiência na escola regular. $\mathrm{Na}$ Grécia, Tafa e Manolitsis (2003) examinaram as atitudes inclusivas de 290 pais de crianças do pré-escolar, revelando que: a) os pais tiveram atitudes positivas; b) quanto maior for o contato com indivíduos com deficiência, menor será a sua preocupação sobre a inclusão de seus filhos; c) as atitudes dos pais não diferem de acordo com seu sexo e seu nível acadêmico;d) os pais parecem reconhecer mais benefícios do que desvantagens para os seus filhos.
Freeman e Alkin (2000) investigaram as atitudes dos pais sobre a socialização e a inclusão dos seus filhos com deficiência e concluíram que mesmo aqueles que acreditavam nos benefícios sociais da inclusão consideraram que os estudantes com deficiência grave seriam rejeitados socialmente. Soodak e Erwin (1995), em estudo realizado em Nova York, entrevistaram nove pais de crianças com deficiência,que relataram suas experiências e percepções sobre a escola.Para esses pais, a filosofia das escolas e as práticas na educação especial não auxiliam convenientemente as metas escolares seus filhos, e, por isso, consideram que deveria haver uma colaboração mais significativa dos educadores com eles.

Gasteiger-Klicpera et al. (2012) analisaram a opinião de 840 pais de crianças de escolas especiais e de turmas inclusivas na Alemanha. Os resultados mostraram que um maior número de pais de crianças que estudavam em classes inclusivas estava satisfeito com a escolaridade dos filhos. To, Kan e Chan (2012), com base numa análise qualitativa de um grupo de pais que participaram num programa de educação em Hong Kong, sugerem que a mudança de atitudes pode ser conseguida se houver esclarecimento deles sobre a importância de se envolverem no processo de inclusão escolar.

\section{Educação Física inclusiva}

A atividade física é definida, segundo Caspersen, Powell e Christerson (1985), como qualquer movimento corporal produzido pelos músculos que resulta em gasto energético. Os autores referem-se ao ato planejado de exercitar-se com o objetivo de melhorar a aptidão física. Para Denise Araújo e Cláudio Araújo, o princípio da inclusão pode também ser entendido como direito de todos à prática de exercício físico e um estilo de vida ativo (2000). Segundo os autores, essa necessidade se ocorre em função de diferentes fatores, entre eles, o fator social, quando se proporciona a essas pessoas o direito de estarem ativas fisicamente em grupo. A EF, como todas áreas de uma sociedade moderna, deve atuar como agente de inserção social de maneira ampla, por intermédio do conhecimento e de ações multidisciplinares.

É importante salientar os avanços que a EF inclusiva tem alcançado ao longo da história, no que se refere à prática de exercício físico adaptado pelas pessoas com deficiência (Winnick, 2010). Considerando a Carta Europeia do Esporte para Todos, que estipula que "todo o indivíduo tem direito à prática desportiva" (Conselho da Europa, 1988, p. 8), também a Educação Física escolar deve ser dirigida e pensada para todos os estudantes, independentemente da sua condição de deficiência. Segundo Soler (2002), o 
principal objetivo da EF com pessoas com necessidades especiais é a sua total reintegração na sociedade.

Em relação às dificuldades de inclusão na disciplina de EF, destaca-se a competição, que por vezes compromete a adaptação dessa matéria às características de cada criança. O problema é maior quando a ênfase dada pelos pais à competição desportiva dos filhos é excessiva, uma vez que muitas vezes pode ser prejudicial ao relacionamento estabelecido entre os estudantes (Garrido et al., 2010). Por outro lado, a falta de conhecimento adequado da comunidade educativa sobre os objetivos e a pertinência das classes de EF leva à sua desvalorização e à falta de entendimento sobre sua aplicabilidade.

A Teoria Crítica da Sociedade, tal como a formularam os pensadores da chamada Escola de Frankfurt, possibilita uma visão crítica da sociedade capitalista, do mundo contemporâneo e da educação. Diante das novas tendências, vive-se o enfraquecimento do sujeito, as desigualdades sociais e a negação, na prática, de direitos fundamentais, como é o caso da prática esportiva. Herbert Marcuse (1982) preocupava-se com o desenvolvimento descontrolado da tecnologia e com o racionalismo dominante nas sociedades modernas, por considerar que reprimiam as liberdades individuais. Ele se opunha a uma sociedade contracultural, por lutar apenas por uma gratificação que a cultura não pode consentir: a gratificação como um fim em si mesma. Adorno (1995) defendeu a educação para a emancipação humana. Segundo o autor, a função do esporte ainda não foi devidamente estudada por uma psicologia social crítica, e a ausência de teoria e de reflexão é uma das suas características, pois, se por um lado, a prática esportiva estimula atitudes de fair-play e respeito pelo mais fraco, por outro, em algumas modalidades, estimula a agressão, a brutalidade e o retrocesso. Jürgen Habermas (1999), um dos herdeiros dessa tradição filosófica, defende uma racionalidade dialógica. Com base no autor, pode-se afirmar que, enquanto no desporto de lazer denomina a racionalidade comunicativa e o respeito pelas diferenças, no esporte institucional, em contrapartida, prevalece a racionalidade instrumental.

Surgem assim algumas contrariedades sobre a prática desportiva das crianças e jovens com deficiência, dentro e fora da escola como é o exemplo também da inacessibilidade de instalações; da falta formação adequada de professores (Martins, 2014; Rodrigues, 2008), bem como a rejeição e o preconceito (Kearney; Kane, 2006).

Por outro lado, a unidade curricular de EF pode constituir um meio favorável para mudar as atitudes em face da população escolar com deficiência, na medida em que pode explorar muitas capacidades desses estudantes, estimulando a cooperação e a solidariedade entre pares, contribuindo assim para a inclusão na comunidade escolar (Martins, 2014; Sherrill, 1998). Torna-se assim importante incentivar os estudantes a valorizar mais a diferença. Esse objetivo pode ser conseguido mediante maior convivência com os seus pares com deficiência, melhores exemplos dos professores e das famílias, e com um clima socioafetivo mais favorável das relações estabelecidas em toda comunidade escolar. No que respeita à atividade física, não podemos nos esquecer da importância da competitividade implícita na prática esportiva, contudo, no que respeita à inclusão de pessoas com deficiência é importante valorizar igualmente o espírito solidário e participativo de todos (Mantoan, 2003).

Muitas vezes os profissionais da escola inclusiva não são devidamente formados para planejar respostas adequadas a todos os alunos, e, por isso, é igualmente importante que psicólogos, terapeutas, médicos, entre outros profissionais, possam dar apoio aos professores de estudantes com deficiência (Martins, 2014; Elkins, Van Kraayenoord, Joblin, 2003). Considera-se, também, que o estabelecimento de uma relação estreita entre a família e a escola confere ênfase ao modo como esses dois contextos interagem e à forma como influenciam as atitudes dos estudantes na inclusão dos colegas com deficiência. Neste sentido, consideramos importante apreciar as relações estabelecidas descrevendo as atitudes parentais em face da inclusão de estudantes com deficiência na unidade curricular de Educação Física.

\section{Metodologia}

Foi realizado um estudo descritivo transversal, de caráter exploratório e enfoque interpretativo. Esta pesquisa foi precedida pela aprovação do Gabinete de Estatística e Planejamento da Educação do Ministério da Educação de Portugal e da autorização dos diretores das escolas-alvo. Participaram 213 pais e tutores legais,encarregados de educação de estudantes que frequentavam escolas do ensino básico do Distrito do Porto, Portugal, no ano letivo de 2011/2012: 83,6\% eram do sexo feminino e $16,4 \%$, do sexo masculino. Com relação à idade, $1,9 \%$ apresentavam menos de 30 anos; 54,9\% tinham 30 a 40 anos; 39,4\% estavam entre os 41 e os 50 anos; e 3,3\% tinham mais de 50 anos. Quanto à formação acadêmica, 47,4\% frequentaram o ensino básico e 30\%, o ensino secundário. Entre aqueles que possuíam estudos superiores, 3,3\% tinham bacharelado; 16,9\%, licenciatura; e 1,4\%, mestrado. Do total, 3,8\% eram pais ou tutores de estudantes com deficiência e 39\% responderam ter relacionamento com pessoas com necessidades especiais. O grupo de discussão foi composto por cinco docentes de EF, três do sexo masculino e dois do sexo feminino, com uma média de idade de 39,4 anos; dois do 
sexo feminino com 39 e 42 anos, duas 2 alunas do $2^{\circ}$ e $3^{\circ}$ Ciclo do EB com 11 e 14 anos, respectivamente.

Para descrever as atitudes sobre a inclusão de estudantes com deficiência em EF, utilizou-se no estudo uma escala de atitudes do tipo Likert "Inventário Geral de Atitudes quanto à EF Inclusiva", desenvolvida no Brasil por Barbosa (2005) e considerada uma ferramenta de mensuração de atitudes em face da educação inclusiva de várias populações (Barbosa; Rosini; Pereira, 2007). A escala mede o grau de concordância dos participantes nas 32 afirmações sobre crenças, sentimentos e tendências de ação perante a inclusão escolar. Metade desses itens diz respeito a afirmações que revelam atitudes positivas, enquanto os restantes medem atitudes negativas, sendo estas cotadas de forma inversa.

Esse instrumento teve ligeiras adaptações em termos de linguagem adaptada à realidade portuguesa e à EF e revelou-se um instrumento com alta consistência interna (Alpha de Cronbach $=0,83$ ), valores que ficaram próximos aos do estudo de Barbosa, Rosini e Pereira (2007), em que obtiveram o valor de 0,87 . A aplicação da escala foi feita durante reuniões de pais no ano letivo 2011/2012. Para analisar a informação recolhida, criou-se uma base de dados na versão 19.0 do programa Statistical Package for the Social Sciences (SPSS). A atitude foi descrita por meio da estatística descritiva e inferencial, (t-test e Anova). O nível de significância foi fixado em $5 \%$.

A tendência mais generalizada na atualidade em estudos de educação é a de utilizar uma metodologia mista combinada os dois enfoques, qualitativo e quantitativo (Pergiorgio, 2003, Reichardt; Cook, 1995). Deste modo e com o objetivo de integrar diferentes perspectivas relativas a fenômeno de estudo,foi aprofundada a discussão a respeito da realidade vivida nas escolas, mediante a abordagem qualitativa. Para tal organizou-se um grupo de discussão online denominado "EF-Inclusiva". Nos últimos anos, essa técnica tem sido usada nas ciências sociais e aconselhada por alguns autores, entre os quais Macnaghten e Myers (2004) e Vicsek (2007). A composição do grupo foi feita de modo intencional e de acordo com critérios de representatividade de professores de EF, pais e alunos. Realizou-se uma análise de conteúdo da informação obtida, que foi organizada em torno dos dois principais descritores: a realidade educativa das classes de EF e as linhas de ação de melhoria.

\section{Apresentação e discussão dos resultados}

A variável atitude inclusiva apresentou uma distribuição normal no teste Kolmogorov-Smir- nov $(0,844 ; p=0,474)$.O valor médio obtido foi de 3,61 $\pm 0,46$ na escala de 1 a 5 , verificando-se no geral que a atitude em relação à EF Inclusiva foi positiva(Quadro1).

Quadro 1 - Análise Descritiva da Atitude Parental Inclusiva

\begin{tabular}{|c|c|c|c|c|c|}
\hline & N & Mín & Máx & M & SD \\
\hline Atitude Global & 213 & 2,47 & 4,69 & 3,61 & 0,465 \\
\hline
\end{tabular}

Legenda: $\mathbf{M}=$ Media; $\mathbf{S D}=$ Standard Deviation Fonte: Elaborado pelas autoras

Pela análise detalhada dos resultados da escala aplicada, os pais e tutores apresentaram atitudes positivas diante do trabalho dos profissionais de EF e pareceram admitir que atualmente pode ser dada a atenção especial de que os alunos com deficiência necessitam. Consideraram que os professores estavam comprometidos como desempenho e a inclusão de todos os alunos nas classes. Também avaliaram positivamente os progressos alcançados nas atitudes sociais dos alunos.

Diante desse resultado, algumas falas dos integrantes do grupo de discussão merecem ser sublinhadas:

Ainda existem pessoas que não aceitam bem os alunos com deficiência, preferiam que eles andassem nas escolas para deficientes. (Estudante 2)

Hoje assistimos a muitos menos preconceitos, mas ainda aparecem pais que dizem: 'Acho muito bem, mas na turma do meu filho, não’. (Tutor 2)

É difícil aceitar a diferença, mas entendê-la ainda é mais complexo. [...] cabe a cada comunidade educativa trabalhar essas questões. (Professor 4)

De acordo com Mantoan (2003), as escolas de qualidade são entendidas como espaços educativos de construção de personalidades humanas autônomas e críticas. Num ambiente educativo alargado à família, deve-se ensinar os alunos a valorizar a diferença pela convivência com os seus pares, pelo exemplo dos professores, pelo ensino ministrado nas classes e pelo clima socioafetivo das relações estabelecidas em toda comunidade escolar, com espírito solidário. Também Ainscow (2002)pontua que, por se caracterizar como um fato existencial, a educação torna-se, obrigatoriamente, uma conjuntura cultural e social.

Para Sanches e Oliveira (2011), os significados e representações sobre as pessoas com necessidades especiais podem não ser compartilhados igualmente por todas as famílias, o que resulta em diferentes atitudes e vivências diante dos problemas, pois cada 
grupo familiar possui representações e valores diferenciados. Muitas dessas representações são determinadas historicamente, enquanto outras recebem a influência de outros contextos, como a religiosidade, a cultura e outros.

Os resultados indicaram médias positivas (na escala de 1 a 5), sugerindo que os tutores manifestaram concordância sobre as possibilidades da Educação Física na inclusão de alunos com deficiência (Quadro 2).

Quadro 2 - Atitudes parentais sobre os aspetos da unidade curricular de EF para facilitar a inclusão

\begin{tabular}{|l|c|c|}
\hline $\begin{array}{l}\text { Efeitos da EF Inclusiva para os pais dos } \\
\text { estudantes com deficiência }\end{array}$ & M & SD \\
\hline $\begin{array}{l}\text { Em EF deve-se dar a esses estudantes to- } \\
\text { das as oportunidades para se incluírem na } \\
\text { classe regular }\end{array}$ & 4,39 & 0,96 \\
\hline $\begin{array}{l}\text { É possível ensinar estudantes com } \\
\text { deficiência e os pares na mesma classe de } \\
\text { EF }\end{array}$ & 4,04 & 1,03 \\
\hline $\begin{array}{l}\text { Os professores de EF regulares não aceitam } \\
\text { bem os estudantes com deficiência }\end{array}$ & 3,65 & 1,27 \\
\hline $\begin{array}{l}\text { Os professores de EF regular jamais terão } \\
\text { preparação suficiente para ensinar estudan- } \\
\text { tes com deficiência }\end{array}$ & 3,29 & 1,40 \\
\hline $\begin{array}{l}\text { Os professores de EF não saberão como li- } \\
\text { dar com preconceitos contra os estudantes } \\
\text { com deficiência }\end{array}$ & 3,29 & 1,29 \\
\hline $\begin{array}{l}\text { É dificil manter a disciplina numa classe de } \\
\text { EF que tenha estudantes com deficiência }\end{array}$ & 3,09 & 1,27 \\
\hline $\begin{array}{l}\text { O rendimento de uma aula de EF é in- } \\
\text { ferior se tiver estudantes com deficiência }\end{array}$ & 2,88 & 1,26 \\
\hline $\begin{array}{l}\text { Educar estudantes com deficiência requer } \\
\text { apoios que não podem ser fornecidos na } \\
\text { EF regular }\end{array}$ & 2,75 & 1,26 \\
\hline $\begin{array}{l}\text { O currículo da EF mesmo com adequa- } \\
\text { cões não atende as necessidades dos estu- } \\
\text { dantes com deficiência }\end{array}$ & 2,60 & 1,09 \\
\hline $\begin{array}{l}\text { As necessidades destes estudantes são } \\
\text { melhor atendidas em aulas de EF especiais } \\
\text { que nas regulares }\end{array}$ & 2,35 & 1,23 \\
\hline
\end{tabular}

Legenda: M: Media; SD: Standard Deviation

Fonte: Elaborado pelas autoras

Considerando a educação como o resultado de uma interação mediante vivências individuais ou coletivas do indivíduo com o meio, a reorganização e reflexão consciente da experiência também está contida no mesmo processo. Segundo Alexandre Baiocchi e Dileuza Baiocchi (2012), o seu grande objetivo será portanto transformar o homem num ser integral, capaz de se elevar através da experiência, do caráter e da personalidade social.

Nesse estudo foram reconhecidas diversas vantagens da inclusão para os alunos com deficiência, para seus pais e para os colegas dos estudantes com deficiência, como se pode verificar pelos resultados apresentados nos Quadros 3, 4 e 5.

Quadro 3 - Atitudes parentais sobre as vantagens da EF para os estudantes com deficiência

\begin{tabular}{|c|c|c|}
\hline $\begin{array}{l}\text { Efeitos da EF Inclusiva para os estudantes } \\
\text { com deficiência }\end{array}$ & $\mathbf{M}$ & SD \\
\hline $\begin{array}{l}\text { Os estudantes com deficiência têm o direito de } \\
\text { serem educados na escola como os seus pares }\end{array}$ & 4,25 & 1,11 \\
\hline $\begin{array}{l}\text { As crianças e jovens com deficiência são mais } \\
\text { felizes quando frequentam aulas de EF com os } \\
\text { pares }\end{array}$ & 4,16 & 1,03 \\
\hline $\begin{array}{l}\text { Estar numa aula de EF regular estimula o desen- } \\
\text { volvimento acadêmico dos estudantes com de- } \\
\text { ficiência }\end{array}$ & 4,16 & ,992 \\
\hline $\begin{array}{l}\text { A maioria dos estudantes com deficiência esfor- } \\
\text { ça-se para completar as atividades nas aulas de EF }\end{array}$ & 4,06 & 1,02 \\
\hline $\begin{array}{l}\text { A inclusão tem um efeito positivo sobre o desen- } \\
\text { volvimento social dos estudantes com deficiência }\end{array}$ & 4,06 & ,95 \\
\hline $\begin{array}{l}\text { A inclusão escolar nas aulas de EF é socialmente } \\
\text { vantajosa para os estudantes com deficiência }\end{array}$ & 4,04 & 1,06 \\
\hline $\begin{array}{l}\text { Estes estudantes desenvolvem mais a autoestima e } \\
\text { o autoconceito na prática de EF regular }\end{array}$ & 3,83 & 1,06 \\
\hline $\begin{array}{l}\text { É provável que a EF Inclusiva prejudique o de- } \\
\text { senvolvimento emocional dos alunos com defi- } \\
\text { ciência }\end{array}$ & 3,75 & 1,30 \\
\hline $\begin{array}{l}\text { Os estudantes com deficiência são socialmente } \\
\text { aceites pelos pares nas aulas de EF }\end{array}$ & 3,72 & 1,01 \\
\hline $\begin{array}{l}\text { A melhor forma de educar os estudantes com } \\
\text { deficiência é simplesmente colocando-os na } \\
\text { escola regular e não esperando que as condições } \\
\text { necessárias estejam presentes }\end{array}$ & 3,57 & 1,45 \\
\hline $\begin{array}{l}\text { É provável que estudantes com deficiência criem } \\
\text { confusão nas aulas de EF }\end{array}$ & 3,12 & 1,27 \\
\hline $\begin{array}{l}\text { Os estudantes com deficiência têm mais dificul- } \\
\text { dades para ter amigos na escola }\end{array}$ & 2,64 & 1,31 \\
\hline
\end{tabular}

Legenda: $\mathbf{M}=$ Media; $\mathbf{S D}=$ Standard Deviatio

Fonte: Elaborado pelas autoras

Quadro 4 - Atitudes sobre os efeitos da inclusão para os pais dos estudantes com deficiência

\begin{tabular}{|l|c|c|}
\hline $\begin{array}{l}\text { Efeitos da EF Inclusiva para os pais dos estudan- } \\
\text { tes com deficiência }\end{array}$ & $\mathrm{M}$ & $\mathrm{SD}$ \\
\hline $\begin{array}{l}\text { A inclusão escolar beneficia as famílias dos estu- } \\
\text { dantes portadores de deficiência }\end{array}$ & 3,67 & 1,25 \\
\hline $\begin{array}{l}\text { Os pais destes estudantes não apresentam maio- } \\
\text { res problemas para o professor de EF do que os } \\
\text { outros pais }\end{array}$ & 3,57 & 1,23 \\
\hline $\begin{array}{l}\text { Os pais dos estudantes com deficiência preci- } \\
\text { sam de mais de apoio por parte dos professores } \\
\text { do que os restantes }\end{array}$ & 3,56 & 1,32 \\
\hline $\begin{array}{l}\text { Os pais destes preferem que eles estudem em sa- } \\
\text { las ou escolas especiais em vez da escola regular }\end{array}$ & 2,99 & 1,33 \\
\hline
\end{tabular}

Legenda: $\mathbf{M}=$ Media; $\mathbf{S D}=$ Standard Deviatio

Fonte: Elaborado pelas autoras 
Quadro 5 - Atitudes parentais sobre o efeito da inclusão para os pares

\begin{tabular}{|l|c|c|}
\hline Efeitos da inclusão em EF para os pares & M & SD \\
\hline $\begin{array}{l}\text { A conduta dos estudantes com deficiência nas } \\
\text { aulas de EF é um mau exemplo para os outros } \\
\text { estudantes }\end{array}$ & 4,34 & 1,04 \\
\hline $\begin{array}{l}\text { Se eu fosse aluno, não me importaria de ter um } \\
\text { colega com deficiência na minha aula de EF }\end{array}$ & 4,33 & 1,03 \\
\hline $\begin{array}{l}\text { A presença destes estudantes nas aulas de EF pro- } \\
\text { move a aceitação da sua diferença por parte dos } \\
\text { colegas }\end{array}$ & 4,10 & 1,18 \\
\hline $\begin{array}{l}\text { O contato dos pares com os estudantes com defi- } \\
\text { ciência nas aulas de EF pode ser prejudicial }\end{array}$ & 4,08 & 1,10 \\
\hline $\begin{array}{l}\text { A inclusão dos estudantes com deficiência nas au- } \\
\text { las de EF pode ser benéfica para os pares }\end{array}$ & 3,96 & 1,11 \\
\hline $\begin{array}{l}\text { Os estudantes com deficiência monopolizam o } \\
\text { tempo e a atenção dos professores de EF }\end{array}$ & 3,21 & 1,31 \\
\hline
\end{tabular}

Legenda: $\mathbf{M}=$ Media; $\mathbf{S D}=$ Standard Deviatio

Fonte: Elaborado pelas autoras

Pelos resultados das atitudes parentais, parece que a unidade curricular EF não enfrentará muitas barreiras na inclusão. Não obstante, é preciso considerar o grupo de encarregados de educação que ainda tendem a possuir atitudes desfavoráveis à inclusão escolar em EF. Essas atitudes parecem emergir do preconceito sobre as consequências negativas no rendimento dos alunos sem deficiência.

Para clarificar as questões, alguns estudos (Ruijs; Van Der Veen; Peetsma, 2010; Qi; Há, 2012) compararam os resultados de alunos que estudavam em turmas inclusivas com os restantes e concluíram que não diferem de forma significante quando é prestado o apoio necessário durante as aulas e é aplicado um currículo adaptado.

Em seguida foi realizada uma análise inferencial da atitude inclusiva em função das variáveis independentes: idade, sexo, formação, presença de filho com deficiência e relacionamento com pessoas com necessidades especiais. Em relação à idade, foi o grupo etário com menos de 30 anos que manifestou uma atitude mais inclusiva, enquanto o grupo com mais de 50 anos obteve o valor mais baixo $(p=0,065)$. Foi também o grupo com idade inferior a 30 anos oque mais valorizou os benefícios da EF inclusiva no desenvolvimento social dos alunos com deficiência e para as suas famílias.

Foi obtida uma diferença significativa em função do sexo $(p=0,007)$, demonstrando que os participantes do sexo feminino concordaram mais $(4,18 \pm 1,09)$ com o fato de o contato com estudantes com deficiência não prejudicar os pares, que os do sexo masculino $(3,63 \pm 1,06)$. No estudo de Barbosa, Rosini e Pereira (2007), as atitudes parentais mostraram-se independentes do sexo, apesar de valores mais positivos para o sexo feminino. Kalyva, Georgiadi e Tsakiris (2007) avaliaram as atitudes inclusivas de 338 pais de crianças da escola primária sem deficiência, e verificaram que os pais manifestaram atitudes mais positivas do que as mães. No entanto, as mães mostraram-se mais interessadas em estimular a interação do seu filho com crianças com deficiência.

Os resultados também demonstraram não haver influência da variável formação acadêmica $(p=0,282)$. Os pais dos alunos portadores de deficiência foram os que apresentaram uma atitude mais positiva e que reconheceram mais as vantagens da EF no desenvolvimento social dos seus filhos $(p=0,022)$. Outros autores, como Barbosa, Rosini e Pereira (2007), Flewitt e Nind (2007), e Leyser e Kirk (2004), também demonstraram que os pais de crianças portadoras de deficiência possuem atitudes mais favoráveis. O papel das famílias desses alunos tem sido considerado, ao longo da história, fundamental para a luta pelos direitos educativos. É sabido que frequentemente essas famílias vivem angústia e estresse intensos relativamente ao futuro, uma vez que essas crianças são o centro das preocupações emocionais e práticas da família (Franco; Apolónico, 2002; Franco, 2011; Stoiber; Gettinger; Goetz, 1998).

Semelhantemente, Leyser e Kirk (2004) concluíram que os pais de alunos com deficiência concordam com a inclusão, sob o ponto de vista jurídico e filosófico, mas se mostraram preocupados com o isolamento social, as atitudes negativas, a qualidade do ensino, a formação de professores, as habilidades, e o apoio de professores e de outros pais. Tanto o Tutor 3 como o Tutor 5 afirmaram que os estudantes que convivem com crianças com várias problemáticas têm mais compreensão e tolerância com a diferença.

O grupo que indicou se relacionar com pessoas com necessidades especiais, concordou mais $(4,59 \pm 0,75)$ que os demais $(4,26 \pm 1,06)$ que as classes de EF devem conceder todas as oportunidades necessárias à participação efetivas desses alunos $(\mathrm{p}=0,038)$. Segundo Franco (2011), a inclusão deve estar assente numa perspectiva de pessoa diferente e no contexto de uma sociedade em mudança que pode pensar a diferença como enriquecedora. Mas essa diferença, acentua o autor, continua em alguns casos a afetar seriamente o funcionamento social de muitos dos seus membros. Como se sabe, a deficiência suscita muitas vezes dimensões emocionais ameaçadoras, relativas à imperfeição.

As vantagens do relacionamento dos alunos com colegas com deficiência também foram valorizadas no grupo de discussão:

As crianças que convivem com a diferença se tornam seres humanos melhores, mais tolerantes... As crianças com deficiência têm uma maior interação social quando convivem com os pares sem deficiência. (Tutor 1) 
Diante desses resultados, os encarregados da educação parecem estar relativamente sensibilizados para a educação inclusiva. De acordo com Glat (2004), os pais podem influenciar positivamente os filhos para o desenvolvimento de suas capacidades e valores sociais. Porém, Silva e Hall (2000) afirmam que a perspectiva da diversidade e da diferença tende a ser naturalizada. Em geral, a posição socialmente aceita é a de tolerância e de respeito à diversidade e à diferença.

Em relação às principais dificuldades de inclusão em EF, o grupo de discussão destacou as seguintes:

- algum preconceito;

- falta de materiais adaptados nas escolas;

- necessidade de formação dos docentes;

- pouca colaboração entre professores e técnicos;

- currículo de EF pouco flexível;

- aulas muito orientadas aos resultados;

- elevado número de estudantes nas classes.

Para o sucesso da EF Inclusiva, foi realçada a melhoria dos seguintes aspectos:

- formação específica dos docentes em deficiência e modalidades desportivas adaptadas;

- condições físicas e materiais didáticos das escolas;

- apoio técnico;

- trabalho colaborativo com as famílias;

- diferenciação do currículo de EF;

- sensibilização dos pares e da comunidade educativa.

O exercício físico é uma área de desenvolvimento muito valorizada pelas famílias, pois o envolvimento das crianças também moldado pelos seus interesses e atitudes (MacDonald et al., 2004). Neste sentido, Muir e Goldblatt (2011) sugerem que os governos precisam fornecer mais o apoio às famílias e à comunidade para assegurar o cumprimento dos direitos humanos das crianças portadoras de deficiência. Ainscow (1997) pontua que um fator igualmente decisivo para a modificação das escolas consiste no envolvimento, que se estende para além da equipe pedagógica e abrange os alunos, os pais e os demais membros da comunidade educativa.

De acordo com Silva e Hall (2000), as questões do multiculturalismo e da diferença tornaram-se, nos últimos anos, centrais na teoria educacional crítica e até mesmo nas pedagogias oficiais. Segundo os autores, é por meio da representação que a identidade e a diferença se ligam aos sistemas de poder, pelo que a pedagogia e o currículo deveriam oferecer oportunidades para que as crianças e jovens desenvolvessem capacidade de crítica dos sistemas e das formas dominantes de representação da identidade e da diferença. Para Sassaki (2007), se os sistemas comuns da sociedade adotarem a inclusão, o quanto antes se verá uma sociedade inclusiva e é nesta que reside o grande desafio social.

\section{Considerações finais}

A respeito das atitudes parentais, a disciplina de EF parece não enfrentar muitas barreiras para a inclusão, e são os pais dos alunos portadores de deficiência os que mais reconhecem as vantagens da EF inclusiva no desenvolvimento social.

Para a formação de atitudes positivas é necessário que as famílias e a comunidade acreditem mais na competência das escolas, e, para tal, são necessárias mais diretrizes do Estado para a melhoria das condições das escolas em termos de formação específica e disponibilização de mais recursos materiais e humanos.

O fator do contato e da experiência com pessoas com deficiência revelou-se como um importante facilitador de atitudes inclusivas. É importante uma colaboração eficaz da escola com as famílias e a comunidade, que possibilite a compreensão mútua e a tolerância dentro e fora da escola, contribuindo assim para um contexto inclusivo mais amplo.

\section{Referências}

ADORNO, Theodor W. Educação e Emancipação. 4. ed. Rio de Janeiro: Paz e Terra, 1995.

AINSCOW, Mel. Educação para todos: torná-la uma realidade. Caminhos para as escolas inclusivas. In: CONGRESSO INTERNACIONAL DE EDUCAÇÃO ESPECIAL, Anais... Birmingham, p.11-31,1997.

. Rutas para el desarrollo de prácticas inclusivas en los sistemas educativos. Revista de Educación, n. 327, p. 69-82, ene./abr. 2002.

ARAÚJO, Denise Sardinha Mendes Soares de; ARAÚJO, Claudio Gil Soares de. Aptidão física, saúde e qualidade de vida relacionada à saúde em adultos. Revista Brasileira de Medicina do Esporte, v. 6, n. 3, p. 194-203, 2000.

BAIOCCHI, Alexandre; BAIOCCHI, Dileuza Niebielski. Educação e inclusão: uma relação de alteridade. Psicopedagogia Online, 2012. ISSN 1808-6225. Disponível em: <http://www. psicopedagogia. com.br/new1_artigo. asp?entrID=1454>. Acesso em: 18 abr. 2013.

BARBOSA, Altemir José Gonçalves. Inventário geral de atitudes quanto à educação inclusiva - IGAEI. São Paulo: Edição do Autor, 2005.

BARBOSA, Altemir José Gonçalves; ROSINI, Daniella 
Collado; PEREIRA, Alessandra Almeida. Atitudes parentais em relação à educação inclusiva. Revista Brasileira de Educação Especial, v. 13, n. 3, p. 447-458, 2007.

BECKETT, Angharad. Challenging disabling attitudes, building an inclusive society: considering the role of education in encouraging non-disabled children to develop positive attitudes towards disabled people. British Journal of Sociology of Education, v. 30, n. 3. p. 317-329, 30 abr. 2009.

BLOCK, Martin; OBRUSNIKOVA, Iva. Inclusion in Physical Education: A Review of the Literature from 1995-2005. Adapted Physical Activity Quarterly, v. 24, n. 2, p. 103-124, abr. 2007.

BOER, Anke; PIJL, Sip Jan; POST, Wendy; MINNAERT, Alexander. Which variables relate to the attitudes of teachers, parents and peers towards students with special educational needs in regular education? Educational Studies, v. 38, n.4, p. 433-448, oct. 2012.

CASPERSEN, Carl J.; POWELL, Kenneth E.; CHRISTERSON, Gregory M. Physical Activity, Exercise and Physical Fitness: Definitions and Distinctions for Health-Related Research. Public Health Reports. v. 100, n. 2, p. 126-131, 1985.

CONSELHO DA EUROPA. Carta europeia do desporto para todos: As pessoas deficientes. Desporto e Sociedade - Antologia de Textos, nº105. Lisboa: Direcção Geral dos Desportos, 1998.

CORREIA, Luís de Miranda; SERRANO, Ana Maria. Envolvimento parental na educação do aluno com NEE. Porto: Porto Editora ed., p. 143-158, 1997.

; Parcerias pais-professores na educação da criança com NEE. In: CORREIA, L. M. (Org.). Inclusão e necessidades educativas Especiais. Porto: Porto Editora, 2003. ELKINS, John; VAN KRAAYENOORD, Christina; JOBLIN, Anne. Parents' attitudes to inclusion of their children with special needs. Journal of Research in Special Educational Needs, v. 3, n. 2, p. 122-129, 2 jul. 2003.

FLEWITT, Rosie; NIND, Melanie. Parents choosing to combine special and inclusive early years settings: The best of both worlds? European Journal of Special Education Needs, v. 22, n. 4, p. 425-441, 12 oct. 2007.

FRANCO, Vítor. A inclusão começa em casa. In: RODRIGUES, David. Educação inclusiva: dos conceitos às práticas de formação. Lisboa: Instituto Piaget, 2011. p. 157-170.

FRANCO, Vítor; APOLÓNICO, Ana María. Desenvolvimento, resiliência e necessidades das famílias com crianças deficientes. Ciência Psicológica, n. 8, p. 40-54, 2002.

FREEMAN, Stephanny; ALKIN, Marvin. Academic and social attainments of children with mental retardation in general education and special education settings. Remedial and Special Education, v. 21, n. 1, p. 3-26, jan. 2000.

GARRIDO, Maria Encarnación; ZAGALAZ, María Luisa; TORRES LUQUE, Gema; ROMERO GRANADOS, Santiago. Diseño y validación de un cuestionario para técnicos deportivos acerca de su opinión sobre las actitudes de padres y madres en el deporte. Cuadernos de Psicología del Deporte, v. 10, n. 2, p. 7-22, jul/dic. 2010. GASTEIGER-KLICPERA, Barbara; KLICPERA, Christian; GEBHARDT, Markus; SCHWAB, Susanne. Attitudes and experiences of parents regarding inclusive and special school education for children with learning and intellectual disabilities. International Journal of Inclusive Education, p. 1-19, 27 jul. 2012.

GLAT, Rosana. Uma família presente e participativa: o papel da família no desenvolvimento e inclusão social da pessoa com necessidades especiais. In: $9^{\circ}$ CONGRESSO ESTADUAL DAS APAEs DE MINAS GERAIS. Anais... Belo Horizonte, 2004.

GÖRANSSON, Kerstin; NILHOLM, Claes; KARLSSON, Kristina. Inclusive education in Sweden? A critical analysis. International Journal of Inclusive Education, $\mathrm{v}$. 15, n. 5, p. 541-555, $1^{\circ}$ jun. 2011.

HABERMAS, Jürgen. Teoría de la acción comunicativa I: racionalidad de la acción y racionalización social. Madrid: Taurus, 1999.

HAYCOCK, David; SMITH, Andy. Inclusive physical education? A study of the management of national curriculum physical education and unplanned outcomes in England. British Journal of Sociology of Education, v. 31, n. 3, p. 291-305, 19 apr. 2010.

HUTZLER, Yeshayahu; LEVI, Inbal. Including children with disability in physical education: general and specific attitudes of high-school students. European Journal of Adapted Physical Activity, v. 1, n. 2, p. 21-30, 2008.

KALYVA, Efrosini; GEORGIADI, Maria; TSAKIRIS, Vlastaris. Attitudes of Greek parents of primary school children without special educational needs to inclusion. European Journal of Special Needs Education, v. 22, n. 4, p. 367-389, 5 jul. 2007.

KEARNEY, Alison; KANE, Ruth. Inclusive education policy in New Zealand: reality or ruse? International Journal of Inclusive Education, v. 10, n. 2-3, p. 201-219, 30 jun. 2006. LIEBERMAN, Lauren; JAMES, Alisa; LUDWA, Nicole. The impact of inclusion in general physical education for all students. Journal of Physical Education, Recreation \& Dance, v. 75, n. 5, p. 37-41, may/jun. 2004.

LEYSER, Yona; KIRK, Rea. Evaluating inclusion: an examination of parent views and factors influencing their perspectives. International Journal of Disability, Development and Education, v. 51, n. 3, p. 271-285, 3 sep. 2004.

MACDONALD, Doune; RODGER, Sylvia; ZIVIANI, Jenny; JENKINS, David; BATCH, Jenny; JONES, Judy. Physical activity as a dimension of family life for lower primary school children. Sport, Education and Society, v. 9, n. 3, p. 307-325, nov. 2004.

MACNAGHTEN, Phil; MYERS, Greg. Focus group. In: SEALE, C.; GOBO, G.; GUBRIUM, J.; Silverman,D. (Eds.).Qualitative research practice. London: Sage, p. 66-79, 2004.

MANTOAN, Maria Eglér. Inclusão escolar: O que é? Por Quê? Como Fazer? São Paulo: Moderna, 2003. (Coleção Cotidiano Escolar). 
Pela escola inclusiva para todos. Direcional Escolas, v. 3, n. 30, jul. 2007.

MARCUSE, Herbert. A ideologia da sociedade unidimensional: o homem unidimensional. 6. ed. Rio de Janeiro: Zahar, 1982.

MARTINS, Celina Luísa Raimundo. Educação física inclusiva: Atitudes dos docentes. Movimento (ESEF/ UFRGS), v. 20, n. 2, p. 637-657, abr./jun. 2014.

MELMAN, Jonas. Família e doença mental: repensando a relação entre profissionais de saúde e familiares. São Paulo: Escrituras Editora, 2001.

MILES, Susie; SINGAL, Nidhi. The education for all and inclusive education debate: conflict, contradiction or opportunity? International Journal of Inclusive Education, v. 14, n. 1, p. 1-15, $1^{\circ}$ feb. 2010.

MORTIER, Kathleen; HUNT, Pam; LEROY, Mieke; VAN de PUTTE, Inge; VAN HOVE, Geert. Communities of practice in inclusive education. Educational Studies, v. 36, n. 3, p. 345-355, 21 jan. 2010.

MUIR, Kristy; GOLDBLATT, Beth. Complementing or conflicting human rights conventions? Realising an inclusive approach to families with a young person with a disability and challenging behaviour. Disability \& Society, v. 26, n. 5 , p. $629-642,1^{\circ}$ aug. 2011.

OMS.Organization World Health (Ed.). International classification of functioning, disability and health: ICF. Geneva: World Health Organization, 2007.

ORSATI, Fernanda T.; CAUSTON-THEOHARIS, Julie. Challenging control: inclusive teachers' and teaching assistants' discourse on students with challenging behaviour. International Journal of Inclusive Education, v. 1, n. 19, 31 may 2012.

PEREIRA-SILVA, Nara Liana; DESSEN, Maria Auxiliadora. Children with and without Down syndrome: parental and teacher values and beliefs. Revista Brasileira de Educação Especial, v. 13, n. 3, p. 429-446, set./dez. 2007.

PERGIORGIO, Corbetta. Metodología y técnicas de investigación social. Madrid: McGraw-Hill, Interamericana de España, SA, 2003.

PORTUGAL. Decreto-Lei n ${ }^{\circ}$ 3, de 7 de janeiro 2008. Diário da República, I série, n. 4. Lisboa: Ministério da Educação, 2008.

QI, Jing; HA, Amy S. Inclusion in Physical Education: A review of literature. International Journal of Disability, Development and Education, v. 59, n. 3, p. 257-281, 9 aug. 2012.

REICHARDT, Ch; COOK, Thomas. Métodos cualitativos $y$ cuantitativos en investigación evaluativa. Madrid: Morata, 1995.

ROCHA, Maria Angélica Moreira; CABUSSU, Maria Arminda S. Tutti; SOARES, Vitória Galvão; LUCENA, Rita. Dislexia: atitudes de inclusão. Revista Psicopedagogia, v. 26, n. 80, p. 242-253, 2009.

RODRIGUES, David. A Educação Física perante a Educação Inclusiva: reflexões conceptuais e metodológicas. Revista da Educação Física, UEM, v. 14, n. 1, p.
67-73, 2008.

RUIJS, Nienke; VAN DER VEEN, Ineke; PEETSMA, Thea. Inclusive education and students without special educational needs. Educational Research, v. 52, n. 4, p. 351390, 3 nov. 2010.

SANCHES, Antonio Gonsales; OLIVEIRA, Márcia Ferreira. Educação inclusiva e alunos com transtorno mental: um desafio interdisciplinar. Psicologia, Teoria $e$ Pesquisa, v. 27 n. 4, p. 411-418, out./dez., 2011.

SANCHES, Isabel; TEODORO, Antônio. Em busca de indicadores de educação inclusiva: o que os professores de apoio educativo dizem que gostariam de fazer. Revista Espaço Pedagógico, v. 17, n. 1, jan./jun. 2012.

SASSAKI, Romeu Kasumi. O direito à educação inclusiva, segundo a ONU. A Convenção sobre os Direitos das Pessoas com Deficiência Comentada. Brasília: Corde, 2007.

SHERRILL, Claudine. Adapted physical activity, recreation and sport: crossdisciplinary and lifespan. Boston: McGrawHill, 1998.

SILVA, Tomaz Tadeu; HALL, Stuart. Identidade e diferença: a perspectiva dos estudos culturais. Petrópolis, RJ: Vozes, 2000.

SINGAL, Nidhi. Inclusive education in India: international concept, national interpretation. International Journal of Disability, Development and Education, v. 53, n. 3, p. 351-369, sep. 2006.

SOLER, Reinaldo. Brincando e aprendendo na educação física especial: planos de aula. Rio de Janeiro: Sprint, 2002.

SOODAK, Leslie C.; ERWIN, Elizabeth J. Parents, professionals, and inclusive education: a call for collaboration. Journal of Educational and Psychological Consultation, v. 6, n. 3, p. 257-276, 1995.

SOUZA, Priscilla Bellard Mende; SILVA, Simone Souza da Costa; PONTES, Fernando Ramos. A quantas anda a inclusão? A relação entre pais e professoras da Educação Inclusiva. Pará: Editora da Universidade do Estado do Pará, 2011. p. 43-50.

SPREAFICO, Andrea. O que quer dizer "integração" nas sociedades de imigração? Sociedade e Cultura, v. 12, n. 1, p. 127-138, 2009.

STOIBER, Karen Callan; GETTINGER, Maribeth; GOETZ, Donna. Exploring Factors Influencing Parents' and Early Childhood Practitioners' Beliefs About Inclusion. Early Childhood Research Quarterly, v. 13, n. 1, p. 107-124, 24 mar. 1998.

SWART, Estelle; ENGELBRECHT, Petra; ELOFF, Raine; OSWALD, Marietjie. Developing inclusive school communities: voices of parents of children with disabilities. Education as Change, v. 8, n. 1, p. 80-108, 2004. TAFA, Eufimia; MANOLITSIS, George. Atitudes de pais gregos de crianças com desenvolvimento típico de jardim de infância em relação à educação inclusiva. European Journal of Special Education Needs, v. 18, n. 2, p. 155-171, 2 jun. 2003.

TO, Siu-ming; KAN, Kcon-wah; CHAN, Ting-sam. A qualitative Analysis of Parents' Perceived Outcomes and 
Experiences in a Parent Education Program Adopting a Transformative Approach. Journal of Social Work Practice, v. 27, n. 1, p. 79-94, 5 out. 2012.

TRIPP, April; RIZZO, Terry; WEBBERT, Linda. Inclusion in physical education: changing the culture. Journal of Physical Education, Recreation E Dance, v. 78, n. 2, p. 32-48, $1^{\circ}$ fev. 2007.

UNESCO. Sobre princípios, políticas e práticas na área das necessidades educativas especiais. Salamanca/Espanha:
UNESCO, 1994.

. Salamanca: dez anos depois. Enabling Education, U.K., 2004. (Edição especial).

VICSEK, Lilla. A scheme for analyzing the results of focus groups. International Journal of Qualitative Methods, v. 6, n. 4, p. 20-34, 5 apr. 2007.

WINNICK, Joseph. Adapted physical education and sport. United Kingdom: Human Kinetics Publishers, 2010.

\title{
Parents' Attitudes to Inclusive Physical Education
}

\begin{abstract}
The attitudes toward inclusion of students with disabilities in regular schools assume a decisive role in building a democratic and inclusive society. The objective of this study was to describe parental attitudes about inclusion in physical education (PE) classes. It was applied a range of social attitudes in a sample of 213 parents or legal guardians of Portuguese students. It was held a discussion group with five PE teachers, two students and two tutors. The results evidenced positive attitudes, which are associated with different factors. We concluded that the success of PE Inclusive requires that families and the community should believe more in the competence of schools. Improvements are needed in human and physical conditions, specific formation, technical support and cooperative work with families and community.
\end{abstract}

Key words: attitudes, parents, inclusive education, physical education, special educational needs.

\section{Actitudes parentales hacia la Educación Física Inclusiva}

\section{Resumen}

Las actitudes hacia la inclusión de los estudiantes con discapacidad en las escuelas regulares tienen un papel decisivo en la construcción de una sociedad democrática e inclusiva. El objetivo de este estudio fue describir las actitudes de los tutores acerca de la inclusión de estudiantes con Necesidades Educativas Especiales (NEE) en las clases de Educación Física (EF). Se aplicó una escala de actitudes sociales sobre EF Inclusiva a una muestra de 213 tutores legales de estudiantes portugueses. También se formó un grupo de discusión con cinco profesores de EF, dos estudiantes y dos tutores. Los resultados mostraron que las actitudes son moderadamente positivas y se relacionan con varios factores. Se concluye que el éxito de la EF Inclusiva requiere que las familias y la comunidad confíen más en la competencia de las escuelas; para ello se necesitan más recursos humanos y materiales; formación específica; la diferenciación de currículo y trabajo colaborativo con las familias y la comunidad.

Palabras clave: actitudes, tutores, educación inclusiva, educación física, necesidades educativas especiales. 